\title{
User-centred food composition data-analysis of user needs through the Use Case approach
}

\author{
M.B. Egan ${ }^{a}$, C. Hodgkins ${ }^{a}$, A. Fragodt ${ }^{a}$, C. Krines $^{b}$, M.M. Raats ${ }^{a}$ \\ ${ }^{a}$ Food Consumer Behaviour and Health Research Centre, Faculty of Arts and Human Sciences, \\ University of Surrey, Guildford, Surrey GU2 7XH, UK \\ ${ }^{\mathrm{b}}$ Technologie-Transfer-Zentrum Bremerhaven (TTZ), Fischai 1, 27572 Bremerhaven, Germany
}

\begin{abstract}
Facilitating access to European food composition data via a web-based system is a key objective of the European Food Information Resource Network of Excellence (EuroFIR). To better understand the needs of potential users of such a system the Use Case approach - a methodology used in software development has been adapted to document the requirements of users. Use Cases describe how a system has to perform in order to be fit for the purpose that it is being used/built for. Several examples of how key users of food composition data currently interact with systems that provide such data have been identified and development of Use Cases will help to determine key functional requirements of the EuroFIR systems being developed and highlight further areas of opportunity for EuroFIR. Facilitating access to enhanced datasets on manufactured foods is one opportunity identified and discussed.
\end{abstract}

\section{Introduction}

Food composition data are used by a wide variety of users in a number of diverse fields (Williamson, 2005). Increasingly there is a need for access to harmonised, comparable, pan-European data in such important areas as dietary intake and health, policy, trade, food industry and agriculture (Egan, Fragodt, Raats, Hodgkins, \& Lumbers, 2007). These areas will require expanded quantitative data on food composition, especially for manufactured foods and ingredients as well as a more integral picture of the foods purchased and consumed within the European market (Krines \& Finglas, 2006). Thus, user requirements and expectations are likely to extend beyond the data currently found and generated in the national food composition databases. To meet these demands one objective of the European Food Information Resource (EuroFIR) project is increased internet availability of European national food composition data and specialised collections through a common access system (Møller, Unwin, Becker, \& Ireland, 2007). Two systems currently being developed within the EuroFIR project to facilitate this access are an E-search system and a database of bioactive compounds (EBasis). An important aspect of this work is the development of a framework for access to and timely incorporation of food industry data into national food composition databases.

The development of a technical system typically encompasses gathering requirements, modelling the system's functions, designing the product, building the system, and using the product. Ivar Jacobson, who worked in the late 1960s on telephony systems at Ericsson, developed the concept of Use Cases to help describe the functional requirements of a system from a user perspective (Jacobson, Christerson, Jonsson, \& Övergaard, 1992). Use cases, essentially a form of text, provide a 
means of modelling and understanding a system's functional requirements based on users' ideas of what they want from the system. By focusing on the system's users the emphasis is on how the system will be used instead of how it will be built or implemented (Cockburn, 2001).

For the purposes of EuroFIR, the 'Use Case' approach has been adapted and employed to identify users' needs when interacting with typical software systems they might use to access and use food composition data as well as some of the key EuroFIR systems currently in development.

Development of Use Cases will help to identify the essential functional requirements of these EuroFIR systems and may also help to identify any areas of opportunity for EuroFIR to add value to its systems in situations where the users' needs are not currently being met, for example by facilitating access to data on branded foods. In the first instance Use Cases are being developed as high level processes to help prioritise the key activity steps that EuroFIR would potentially satisfy; subsequently the relevant Use Cases will be expanded to detail the exact functional specifications required to satisfy the users.

\section{Key processes for analysis}

The 'Use Case' approach results in a functional requirement analysis for a specified activity within a process whereby a user interacts with a system in order to satisfy a specific goal. Selection of the most relevant high level processes for analysis is a key step. It is important to remember that a number of users from different disciplines may undertake the same process but have differing needs of a system. Therefore in order to obtain a complete set of functional requirements for the system it may be necessary to develop several Use Case write-ups for any one process, depending on how many different types of users there are. Overlaying the results then allows identification of the functionality that will satisfy the widest group of users.

Within the area of food composition there are a vast number of processes performed where users interact with database systems or potentially will interact with the EuroFIR systems, either directly or indirectly. Similarly, there are a vast number of different types of users. For the purposes of this work it is necessary to focus on a number of key processes and an associated set of appropriate primary users from which it is felt that the most useful data for EuroFIR will arise.

Examples of processes that rely on food composition data and their associated primary users have been identified and are listed in Table 1. One of these processes will be presented in more detail as an example. Writing a Use Case involves the use of certain specific terms and these are explained here.

A process typically describes a single goal and all the possible things that can happen as a user attempts to reach that goal. A primary user initiates interaction with the system and has a goal with respect to that system. The overall process typically contains more than one activity step. A Use Case write-up is developed for a particular activity which contains a sequence of action steps. A successful flow of events details the sequence of action steps within the chosen activity which result in all the user's functional requirements being met.

Inevitably within any activity, some of the action steps end with success; some end with failure or more likely result in the user needing to employ a 'coping strategy' by branching away from the 
successful flow in order to satisfy their needs. It is these exceptions or alternative flows that clearly identify where the user's needs are not being met by the system employed.

Use Case write-ups will not be developed for all activities within a given process; they focus only on the activity steps within the process where the user is directly interacting with software or another system supplying the food composition data.

\section{Example of a EuroFIR Use Case write-up: dietary analysis for an individual}

A Use Case write-up has been developed for the dietary analysis of an individual person's diet in a non-clinical setting. Several interviews were conducted with a key informant to ensure that the steps in the process are described. Initially a brief description of the overall process was prepared:

'A client records dietary information in a food diary and passes it to a dietitian who checks and clarifies items with the client. The dietitian enters the food diary information into a dietary analysis programme. The programme performs a nutritional analysis based on all data entry and contrasts the results with personalised requirements, e.g. estimated energy requirements. The dietitian produces a report that includes the results and an evaluation of the diet which can include outputs from the analysis programme, e.g. graphs, Dietary Reference Value (DRV) tables, information on safe upper limits. The dietitian discusses the report with the client and makes any necessary recommendations.'

The process was then expanded into a sequence of activity steps in order to highlight the use of food composition data in this example (Table 2). From this sequence it emerges that the primary user interacts most significantly with the food composition data within the software system in activity 4 i.e. the step where the food diary data is entered into the dietary analysis software. This activity is subsequently analysed in terms of a successful flow of events and associated exceptions or alternatives flows (Table 3).

\subsection{Exceptions/alternative flows}

In considering the exceptions documented in this Use Case write-up, a number of potential opportunities for EuroFIR to meet the users' needs can be identified. These needs can be satisfied either by the EuroFIR systems currently under development, or by EuroFIR supporting or supplementing the systems currently employed by the user (Table 4).

\section{Results of the Use Case analysis}

It is apparent from the Use Case analysis of an individual's diet that the majority of the user's needs are being met by the nutritional software employed and generally the user's frustration relates to lack of appropriate food items or nutritional data within the datasets available to them. On the whole the opportunities identified for EuroFIR to meet these needs are already being addressed within the project but this will not necessarily be the case for all of the key processes identified for 
analysis. For example, the health claim substantiation process is a reasonably new requirement due to the recent changes in legislation and it is likely that expansion of this process into potential activity steps and development of Use Case write-ups may identify some key user needs that have yet to be addressed by any systems currently available or indeed within the systems that EuroFIR is developing (i.e. E-Basis).

\subsection{Meeting user needs via data set expansion}

One very important need identified from the Use Case analysis described is the increased availability of and access to more detailed food composition data on a wider range of foods and nutrients (Table 4). This reflects similar needs of US dietitians who report a lack of data for some foods or ingredients in food composition software, with a particular requirement for data on institutional foodservice products, commercial ingredients and nutritional supplements (McCabe-Sellers \& Chenard, 2008). The provision of such detailed information for an increasing number of constantly changing foods and the respective costs for analysis of various food constituents remains a huge challenge and requires ongoing cooperation and the establishment of collaborative systems.

Increasing the availability of food composition data involves the linking of national databases and thus a sharing of relevant data sets between national compilers (e.g. common products, panEuropean formulations). There is a particular need for greater coverage of manufactured and branded foods, as well as relevant ingredients. As these represent the foods actually sold and consumed by the public in the modern market place. Facilitating transfer of data by multinational companies to a single portal rather than multiple compilers is an important step in the process. The establishment of minimum requirements for food composition data provided by industry, retail or industrial organisations will allow for the incorporation of such data in quality-checked, validated national food composition data sets. Ultimately these and other activities will potentially lead to the establishment of a collaborative framework for the transfer of timely and accurate food composition information on manufactured products, which will allow the expansion of available data sets. However this process involves numerous challenges including the number and variety of manufactured foods, technological barriers to data transfer and issues of confidentiality and intellectual property.

\section{Conclusions}

The benefit of the Use Case approach in developing user-centred food composition data lies in the focus on the user and their use of data. A systematic analysis of users needs will inevitably highlight many areas where the users feel that their needs are not being met.

Analysis of this process clearly shows where EuroFIR might potentially 'add value' over the current system employed however it would be unrealistic to suggest that EuroFIR undertakes to resolve all of the user needs identified. Development of a range of Use Case write-ups for the key processes identified will allow EuroFIR to scope out which goals it intends it's systems to satisfy and for which key users or user groups. It is important to manage user expectations given the complex nature of 
activities such as the expansion of industrial data sets and enhanced data transfer which may be limited in providing user satisfaction.

Similarly it is not envisaged that resolution is limited to software development within EuroFIR's core product offering i.e. The Food Composition Databank System, E-Basis, specialised datasets, etc. Many of the exceptions highlighted in this example might best be satisfied by the establishment of collaborative networks and minimum standards for data transfer. In addition the provision of documented procedures, training or technical support that could be used by others developing systems for users would serve to improve and enhance standards across Europe.

\section{Acknowledgements}

This work was completed on behalf of the European Food Information Resource Consortium and funded under the EU 6th Framework Food quality and Safety programme, project number FP6513944.

\section{References}

Cockburn, A. (2001). Writing Effective Use Cases. Addison-Wesley.

Egan, M. B., Fragodt, A., Raats, M. M., Hodgkins, C., \& Lumbers, M. (2007). The importance of harmonizing food composition data across Europe. European Journal of Clinical Nutrition, 61, 813821.

Jacobson, I., Christerson, M., Jonsson, P., \& Övergaard, G. (1992). Object-orientated software engineering: A Use Case driven approach. Information and Software Technology, 34, 825-826.

Krines, C., \& Finglas, P. (2006). The industrial role and perspective in the quest for better nutritional data. Food Science and Technology, 20, 51-56.

McCabe-Sellers, B. J., \& Chenard, C. A. (2008). Meeting the needs of US dietitians for food composition data. Journal of Food Composition and Analysis, 21, S27-S34.

Møller, A., Unwin, I. D., Becker, W., \& Ireland, J. (2007). EuroFIR's food databank systems for nutrients and bioactives. Trends in Food Science and Technology, 18, 428-433.

Williamson, C. (2005). The different uses of food composition databases, Synthesis Report No. 2. Available at URL: www.eurofir.net/UsesspofspFCDBs.pdf. 
Table 1 Key processes that use food composition data and associated primary users

\begin{tabular}{|c|c|}
\hline Key processes & Primary users \\
\hline Dietary analysis for an individual person / group of people & $\begin{array}{l}\text { - } \text { Dietitians } \\
\text { - } \text { Nutritionists } \\
\text { - } \text { Researchers }\end{array}$ \\
\hline Menu cycle development (e.g. in a care home or school) & $\begin{array}{l}\text { - Food service providers / } \\
\text { commissioners } \\
\text { - Public Health nutritionists }\end{array}$ \\
\hline Developing food product labelling & $\begin{array}{ll}\text { - } & \text { Food manufacturers } \\
\text { - } & \text { Food retailers } \\
\text { - } & \text { Food Service providers }\end{array}$ \\
\hline Compilation of a food composition databank & $\begin{array}{ll}\text { - } & \text { Food composition database } \\
\text { - } & \text { Gompilers } \\
\text { - } & \text { Nutrition Survey Managers } \\
\text { - } & \text { Researchers }\end{array}$ \\
\hline Risk analysis & - Food Safety agencies \\
\hline Health Claim Substantiation & $\begin{array}{ll}\text { - } & \text { EFSA/Government/Regulatory } \\
& \text { bodies } \\
\text { - } & \text { Food manufacturers } \\
\text { - } & \text { Researchers }\end{array}$ \\
\hline Nutrient profiling & $\begin{array}{l}\text { - } \text { EFSA/Government/Regulatory } \\
\text { bodies } \\
\text { - Food manufacturers } \\
\text { - } \text { Food Service providers }\end{array}$ \\
\hline
\end{tabular}


Table 2 Activity steps in the dietary analysis for an individual person

\begin{tabular}{|c|c|c|}
\hline Activity & Description & Detail \\
\hline 1 & $\begin{array}{l}\text { Collect dietary } \\
\text { information }\end{array}$ & $\begin{array}{l}\text { - Obtain details of food consumption from client's food diary } \\
\text { (paper or electronic) }\end{array}$ \\
\hline 2 & $\begin{array}{l}\text { Initial checking and } \\
\text { clarification of data }\end{array}$ & $\begin{array}{l}\text { - Check information provided by clientface-to- } \\
\text { face/telephone/email }\end{array}$ \\
\hline 3 & $\begin{array}{l}\text { Coding and portion } \\
\text { size assessment }\end{array}$ & $\begin{array}{l}\text { - Assign codes to foods manually (optional) } \\
\text { - Assess portion sizes and convert to grams using food portion } \\
\text { size book and manufacturer's data (optional) }\end{array}$ \\
\hline 4 & Data entry & - Enter food diary data into dietary analysis programme \\
\hline 5 & Data analysis & $\begin{array}{l}\text { - Analyse diet for macronutrients, micronutrients, energy, other } \\
\text { constituents }\end{array}$ \\
\hline 6 & Report writing & $\begin{array}{l}\text { - Present results in appropriate format for both dietitian and } \\
\text { client, based on original purpose of dietary analysis }\end{array}$ \\
\hline
\end{tabular}


Table 3 Details of Activity 4- Enter food diary data into dietary analysis programme

Primary User: Dietitian

Preconditions (what must be true before use case runs):

Dietitian has access to a dietary analysis programme and to client's food diary.

Goal: Dietary analysis programme provides accurate, up-to-date data for all food items in client's diary.

Successful flow of events (the case in which all the user's functional requirements are satisfied):

1. User enters generic food item name

2. User selects appropriate source of food composition data to search

3. User selects required food item from list of foods that system presents

4. System assigns food code and lists nutrient composition

5. User selects or enters portion size

6. User adds food to per day/ per meal list

7. User repeats steps 1-6 for all food diary data

8. User proceeds to Activity 5 (data analysis - see Table 2)

Exceptions/Alternative flows:

2a. Appropriate food composition data unavailable in programme

Alternative flows:

Exclude food if negligible nutrient content

Replace food with an equivalent food from database

Replace food with an equivalent food from manufacturer's data

Add food to database as an 'extra' food

Add values to database for composite foods/dishes

3a. Food item unavailable

Alternative flows:

Exclude food if negligible nutrient content

Replace food with an equivalent food from database

Replace food with an equivalent food from manufacturer's data

Add food to database as an 'extra' food

Add values to database for composite foods/dishes

3b. Food item available but not appropriate preparation/cooking method

Alternative flows:

Exclude food if negligible nutrient content

Replace food with an equivalent food from database

Replace food with an equivalent food from manufacturer's data

Add food to database as an 'extra' food

Add values to database for composite foods/dishes

3c. Food item available, missing nutrient values

Alternative flows:

Replace food with an equivalent food from database which includes the required nutrient values

Replace food with an equivalent food from manufacturer's data

Add nutrient value to database as an 'extra' nutrient

$5 a$ Information on typical portion size unavailable

Alternative flows:

Identify portion size from an alternative source and enter 
Table 4 Opportunities for EuroFIR to meet user's needs

Exceptions

2a. Appropriate food composition datasets unavailable in programme for selection

3a. Food item unavailable

3b. Food item available but not appropriate preparation / cooking method

3c. Food item available, missing nutrient values
Opportunities for EuroFIR to meet Users' needs

- Provide access to other national food composition datasets, specialised datasets and manufactured foods datasets.

- Provide access to other national food composition datasets, specialised datasets and manufactured foods datasets, thus ensuring access for the user to a wider coverage of foods.

- Extend available datasets to include nutritional data on foods prepared by a wider variety of cooking methods and to manufactured food datasets.

- Provide standardised methodology and tools for calculating nutritional values for prepared foods from raw food data.

- Provide recommended methodology and tools for recipe calculation

- Provide access to a training resources for food composition data management and use

- Extend available datasets to include wider coverage of nutrients 